\title{
Identification of aberrantly expressed long non-coding RNAs in postmenopausal osteoporosis
}

\author{
QI FEI*, XIAODONG BAI*, JISHENG LIN, HAI MENG, YONG YANG and AI GUO \\ Department of Orthopedics, Beijing Friendship Hospital, Capital Medical University, Beijing 100050, P.R. China
}

Received October 19, 2017; Accepted February 14, 2018

DOI: $10.3892 / \mathrm{ijmm} .2018 .3575$

\begin{abstract}
Postmenopausal osteoporosis (PMOP) is a common skeletal disorder in postmenopausal women. The present study aimed to identify the key long non-coding RNAs (lncRNAs) in PMOP through RNA sequencing. RNA sequencing was performed to obtain the expression profile of lncRNAs and mRNAs in blood samples of patients with PMOP and normal controls (NCs). Following the identification of differentially expressed mRNAs (DEmRNAs) and differentially expressed lncRNAs (DElncRNAs), the DElncRNA-DEmRNA co-expression network was constructed. A search was performed for the DEGs transcribed within a 100-kb window upstream or downstream of DElncRNAs, which served as nearby DEmRNAs of DElncRNAs. Functional annotation of the DEmRNAs co-expressed with DElncRNAs was performed. The GSE56815 dataset was used to verify the expression of selected DEmRNAs and DElncRNAs. Three blood samples from patients with PMOP and two blood samples from NCs were used for RNA sequencing. Compared with the NC group, a total of 185 DEmRNAs and 51 DElncRNAs were obtained in PMOP. A total of 3,057 co-expression DElncRNA-DEmRNA pairs and 97 DElncRNA-nearby DEmRNA pairs were obtained. Six DEmRNAs [diacylglycerol O-acyltransferase 2, potassium voltage-gated channel subfamily $\mathrm{S}$ member 1 , peptidase inhibitor 3, secretory leukocyte peptidase inhibitor, galectin-related protein and alkaline phosphatase, liver/bone/kidney (ALPL)] were nearby co-expressed genes of four DElncRNAs, including LOC105376834, LOC101929866, LOC105374771 and LOC100506113. Three PMOP-associated DEmRNAs, including ALPL, suppressor of cytokine signaling 3 and adrenomedullin, were co-expressed with the hub
\end{abstract}

Correspondence to: Dr Qi Fei or Dr Ai Guo, Department of Orthopedics, Beijing Friendship Hospital, Capital Medical University, 95 Yong'an Road, Xicheng, Beijing 100050, P.R. China

E-mail: spinefei@126.com

E-mail: guoaiortho@126.com

*Contributed equally

Key words: long non-coding RNA, RNA sequencing, postmenopausal osteoporosis, co-expression
DElncRNAs (LINC00963, LOC105378415, LOC105377067, HCG27, LOC101928143 and LINC01094) of the positively and negatively co-expressed DElncRNA-DEmRNA interaction network. The expression of selected DEmRNAs and DElncRNAs was consistent with the RNA-sequencing results. In conclusion, the present study identified the key DEmRNAs and DElncRNAs in PMOP, which may provide clues for understanding the mechanism and developing novel biomarkers for PMOP.

\section{Introduction}

Osteoporosis is a systemic skeletal disorder characterized by a reduction in bone mineral density (BMD) and disrupted bone architecture, which results in a higher risk of bone fractures $(1,2)$. It is reported that $\sim 50 \%$ of postmenopausal women suffer from osteoporosis, which is defined as postmenopausal osteoporosis (PMOP) $(3,4)$. The basic pathogenesis of PMOP involves an imbalance between bone resorption by osteoclasts and bone formation by osteoblasts, which is mainly induced by decreased estrogen. As PMOP is a chronic disease, it imposes a significant financial burden on postmenopausal women $(4,5)$. Therefore, it is crucial to uncover the mechanism and develop accurate diagnostic biomarkers of PMOP.

Previous studies have indicated that osteoporosis and BMD are heritable $(6,7)$, and $>60$ susceptible loci have been found to be associated with osteoporosis and BMD (6). Among these, polymorphisms of several genes have been found to be involved in PMOP, including tumor necrosis factor (TNF)- $\alpha$, interleukin (IL)10, osteoprotegerin, estrogen receptor 1 gene, estrogen receptor $\alpha$, cannabinoid receptor 2 , vitamin D receptor gene and LDL receptor related protein 5 (8-14).

Long non-coding RNAs (lncRNAs) are a set of non-coding RNAs containing $>200$ nucleotides. There has been increased interest focused on IncRNAs, which have been found to be involved in diseases, including cancer and osteoporosis by regulating their target genes at the transcriptional, post-transcriptional and epigenetic levels $(15,16)$. An lncRNA, DANCR was found to be involved in PMOP by regulating TNF- $\alpha$ and IL6 (16). LncRNA MEG3 can suppress the osteogenic differentiation of bone marrow mesenchymal stem cells induced by PMOP (17). However, reports of lncRNAs in PMOP remain limited.

In the present study, the IncRNA and mRNA expression profile of blood samples from patients with PMOP and 
normal controls (NCs) were identified by high-throughput RNA-sequencing. To the best of our knowledge, the present study is the first to obtain the lncRNA expression profiles of PMOP by RNA sequencing. Based on the identified differentially expressed lncRNAs (DElncRNAs) and differentially expressed mRNAs (DEmRNAs) in PMOP, compared with NC, the DElncRNAs-DEmRNAs co-expression network was constructed. The potential roles of these DElncRNAs were further examined according to the functional annotation of their co-expressed DEmRNAs. These findings may provide clues for understanding the pathogenesis and novel insight for developing diagnostic biomarkers of PMOP.

\section{Materials and methods}

Patients and samples. From April 2016 to March 2017, three women with PMOP and two healthy women from Beijing Friendship Hospital were enrolled in the present study. The inclusion criteria of patients with PMOP were as follows: i) Postmenopausal women who were diagnosed with osteoporosis. Osteoporosis was defined by the World Health Organization criteria of a BMD T-score of -2.5 standard deviations below the average for a young adult at peak bone density in the femoral neck, total hip, or L1-L4; ii) clinically symptomatic postmenopausal women with painful vertebral fractures verified by X-ray and MRI within the last 6 months, who returned for further examination and treatment. The patient characteristics are listed in Table I. All individuals provided written informed consent for use of their samples in the present study. The present study was approved by the Ethics Committee of Beijing Friendship Hospital, Capital Medical University (Beijing, China; 2017-P2-084-01). From every participant, a $2.5 \mathrm{ml}$ peripheral whole blood was collected in PAXgene ${ }^{\circledR}$ RNA blood tubes (PreAnalytiX $\mathrm{GmbH}$, Hombrechtikon, Switzerland) and stored at $-80^{\circ} \mathrm{C}$ prior to processing.

RNA isolation and sequencing. RNA isolation was performed using the PAXgene blood RNA kit (PreAnalytiX GmbH) according to the manufacturer's protocol. The concentration and purity of RNA were assessed using a Nanodrop ND-2000 spectrophotometer (Thermo Fisher Scientific, Inc., Waltham, MA, USA). The integrity of RNA was assessed using a $2 \%$ agarose gel. A RIN value was obtained using an Agilent 2100 bioanalyzer. The criteria for cDNA library construction were as follows: i) Total RNA $>5 \mu \mathrm{g}$; ii) concentration of RNA $\geq 200 \mathrm{ng} / \mathrm{ml}$; iii) OD260/280 value 1.8-2.2.

Following removal of the ribosomal RNA using the Ribo-Zero Magnetic kit (EpiCentre, Madison, WI, USA), the RNA was purified and fragmented into 200-500-base pair fragments. The RNA fragments were primed with random hexamer primers and the first cDNA strand was synthesized, with the second cDNA strand synthesized with dUTP instead of dTTP. The blunt ends of double-stranded DNA were produced from cohesive ends of double-stranded DNA using End Repair Enzyme mix (New England BioLabs, Inc., Ipswich, MA, USA). Subsequently, 3'end adenylation and adapter ligation were performed. When the second digested cDNA strand was digested using the UNG enzyme (Illumina, Inc., San Diego, CA, USA), polymerase chain reaction (PCR) was performed with PCR Primer Cocktail (Illumina, Inc.) and PCR Master Mix (Illumina, Inc.) to amplify the libraries. The following thermocycling conditions were used for the PCR: Initial denaturation at $98^{\circ} \mathrm{C}$ for $30 \mathrm{sec} ; 15$ cycles of $98^{\circ} \mathrm{C}$ for $10 \mathrm{sec}, 65^{\circ} \mathrm{C}$ for $30 \mathrm{sec}$ and $72^{\circ} \mathrm{C}$ for $30 \mathrm{sec}$, followed by a final extension step of $72^{\circ} \mathrm{C}$ for $5 \mathrm{~min}$. Certified Low Range Ultra Agarose (Bio-Rad Laboratories, Inc., Hercules, CA, USA) was used to purify the libraries, and the libraries were quantified using Picogreen double-stranded DNA quantitation kit (Molecular Probes; Thermo Fisher Scientific, Inc.) on a TBS380 fluorometer (Promega Corporation, Madison, WI, USA). The qualified libraries were amplified on cBot to generate the cluster on the flowcell using TruSeq PE Cluster kit V3-cBot-HS (Illumina, Inc.) according to the manufacturer's protocol. Sequencing was performed on the Illumina Hiseq Xten platform (Illumina, Inc.).

Quality control of raw sequence and mapping of clean reads. The FASTQ sequence data were obtained from the RNA-seq data using Base Calling V0.11.4 (http://www. bioinformatics.babraham.ac.uk/projects/fastqc/). To obtain the high quality clean data, the low quality reads including adaptor sequences, sequences with a quality score $<20$, and sequences with an $\mathrm{N}$ base rate of raw reads $>10 \%$ were removed by using Cutadapt V1.9.1 (https://cutadapt. readthedocs.io/en/stable/). With TopHat release 2.1.1 (http://tophat.cbcb.umd.edu/) and Ensemble gene annotation, clean reads were aligned with the human reference genome, Ensemble GRCh38.p7 (ftp://ftp.ncbi.nlm.nih. gov/genomes/Homo_sapiens). The expression of mRNAs and lncRNAs was determined and outputted using Cuffquant V2.2.1 (http://cufflinks.cbcb.umd.edu/).

Identification of DEmRNAs and DElncRNAs in PMOP compared with $N C$. Fragments per Kilobase of exon per million fragments mapped (FPKM) was used to determine the transcription abundance of lncRNAs and mRNAs. The FPKMs of lncRNAs and mRNAs were calculated using Cuffdiff (http://cole-trapnell-lab.github.io/cufflinks/cuffdiff/index.html). A paired t-test was performed to obtain the DEmRNAs and DElncRNAs in PMOP compared with NC. The thresholds of the DEmRNAs and DElncRNAs was $\mathrm{P}<0.05$.

DElncRNA-DEmRNA co-expression network. To further examine the potential roles of DElncRNAs and DEmRNAs in PMOP the DElncRNA-DEmRNA co-expression network was constructed. Firstly, the Pearson's correlation coefficient (PCC) between the expression levels of each DElncRNA-DEmRNA pair in the PMOP and the NC group were calculated. Secondly, DElncRNA-DEmRNA pairs with an absolute value of $P C C \geq 0.90$ and $P<0.05$ were defined as co-expressed DElncRNA-DEmRNA pairs. Those co-expressed DElncRNA-DEmRNA pairs in which the expression level of DEmRNAs was positively correlated with the expression level of DElncRNAs in PMOP were defined as positively co-expressed DElncRNA-DEmRNA pairs. Co-expressed DElncRNA-DEmRNA pairs in which the expression level of DEmRNAs was negatively correlated with the expression level of DElncRNAs in PMOP were defined as negatively 
Table I. Patient characteristics.

\begin{tabular}{|c|c|c|c|c|c|}
\hline Characteristic & Case 1 & Case 2 & Case 3 & Control 1 & Control 2 \\
\hline Age (years) & 81 & 68 & 79 & 67 & 68 \\
\hline Sex & Female & Female & Female & Female & Female \\
\hline BMI $\left(\mathrm{kg} / \mathrm{m}^{2}\right)$ & 23.1 & 15.6 & 15.6 & 28.6 & 22.5 \\
\hline BMD-T score & -3 & -4.2 & -3.7 & 1.5 & 0.4 \\
\hline History of smoking & No & No & No & No & No \\
\hline History of alcohol intake & No & No & No & No & No \\
\hline History of coffee or carbonated drink intake & No & No & No & No & No \\
\hline Family history of matrilineal family & No & No & No & No & No \\
\hline Lack of physical activity & No & No & No & No & No \\
\hline Bone metabolism-associated disease & No & No & No & No & No \\
\hline Bone metabolism-associated drugs & No & No & No & No & No \\
\hline
\end{tabular}

BMI, body mass index; BMD, bone mineral density.

co-expressed DElncRNA-DEmRNA pairs. The positively and negatively co-expressed DElncRNA-DEmRNA networks were visualized using Cytoscape 3.1 (http://cytoscape.org/).

Nearby DEmRNAs of the DElncRNAs. In order to identify the targeted DEmRNAs of DElncRNAs by cis-regulatory effects, a search was performed for the DEmRNAs transcribed within a $100-\mathrm{kb}$ window upstream or downstream of DElncRNAs, which served as nearby cis-targeted DEmRNAs of DElncRNAs.

Functional annotation of DEmRNAs co-expressed lncRNAs. Functional annotation, including Gene Ontology (GO) function and Kyoto Encyclopedia of Genes and Genomes (KEGG) pathway enrichment analyses of the DEmRNAs co-expressed with DElncRNAs was performed using the GeneCoDis3 tool (http://genecodis.cnb.csic.es/analysis). A hypergeometric test was used to obtain the P-value. The false discovery rate (FDR; corrected P-value) of $<0.05$ was set as the cut-off for significant GO terms and KEGG pathways.

Validation in the Gene Expression Omnibus (GEO) dataset. The GSE56815 dataset was obtained from the GEO (https://www.ncbi.nlm.nih.gov/geo/), which consisted of 20 postmenopausal women with low hip BMD (case group) and 20postmenopausal women with high hip BMD (normal group). All 40 females were Caucasian. The expression pattern of selected DElncRNAs and DEmRNAs was verified using the GSE56815 dataset. GSE7158 was another dataset obtained from GEO, which consisted of 12 women with a low peak bone mass (case group) and 14 women with a high peak bone mass (normal group). GSE7158 was also used to validate the expression pattern of selected DElncRNAs.

\section{Results}

RNA-sequencing data. Total RNA extracted from each of the blood samples met the criteria for cDNA library construction and RNA-sequencing. Following trimming of the raw reads, $6.8 \times 10^{7}, 6.8 \times 10^{7}$ and $6.7 \times 10^{7}$ clean reads were obtained from the three respective blood samples from patients with postmenopausal osteoporosis; $6.8 \times 10^{7}$ and $6.7 \times 10^{7}$ clean reads were obtained from the two respective NCs. All of the clean reads were aligned to the human reference genome (GRCh38.p7) and the mapped ratio of all samples was $>80 \%$.

DEmRNAs and DElncRNAs in PMOP. A total of 185 significantly DEmRNAs (184 upregulated DEmRNAs and one downregulated DEmRNAs) were obtained with $\mathrm{P}<0.05$. The top 30 significant DEmRNAs are listed in Table II. A total of 51 significantly DElncRNAs (25 upregulated DElncRNAs and 26 downregulated DElncRNAs) were obtained with $\mathrm{P}<0.05$ (Table III). LOC105372321 was the most markedly upregulated DElncRNA and LOC105374771 was the most markedly downregulated DElncRNA in PMOP, compared with NC. NOD-like receptor family pyrin domain containing 6 was the most significantly upregulated DEmRNA and PAGE family member 2B was the only downregulated DEmRNA. Furthermore, these DElncRNAs were distributed in all chromosomes (chr.), with the exception of chr. 5, 15, 17, 18, 21 and sex chr. X and Y, whereas the DEmRNAs were widely distributed in all chromosomes, except sex chr.Y (Fig. 1).

DElncRNA-DEmRNA co-expression network. Based on the expression levels of DElncRNAs and DEmRNAs, the PCC describing the co-expression association between 185 DElncRNAs and 51 DEmRNAs, was calculated. A total of 3,057 DElncRNA-DEmRNA co-expression pairs were obtained with an absolute value of PCC $\geq 0.90$ and $\mathrm{P}<0.05$. Among these, a total of 2,756 lncRNA-mRNA pairs were identified as being positively co-expressed, whereas 301 lncRNA-mRNA pairs were negatively co-expressed. The positively co-expressed DElncRNA-DEmRNA network (Fig. 2A) consisted of 215 nodes and 2,756 edges, and its hub lncRNAs were LOC105378415 (degree $=159)$, LOC105377067 (degree=157), HCG27 (degree=157), 
Table II. Top 30 significantly DEGs in patients with postmenopausal osteoporosis compared with normal controls.

\begin{tabular}{|c|c|c|c|c|}
\hline DEG & Locus & $\log 2$ (fold-change) & Regulation & P-value \\
\hline FOLR3 & chr11:72135709-72139892 & -4.03 & Down & $5.00 \times 10^{-05}$ \\
\hline PI3 & chr20:45174898-45176544 & -2.88 & Down & $5.00 \times 10^{-05}$ \\
\hline KRT23 & chr17:40922695-40987135 & -2.76 & Down & $5.00 \times 10^{-05}$ \\
\hline CD177 & chr19:43353658-43366075 & -2.57 & Down & $5.00 \times 10^{-05}$ \\
\hline REM2 & chr14:22883164-22887680 & -2.15 & Down & $5.00 \times 10^{-05}$ \\
\hline NLRP6 & chr11:278364-285942 & -2.14 & Down & $5.00 \times 10^{-05}$ \\
\hline MANSC1 & chr12:12329262-12350541 & -2.06 & Down & $1.00 \times 10^{-04}$ \\
\hline LRG1 & chr19:4537214-4540024 & -2.14 & Down & $1.50 \times 10^{-04}$ \\
\hline HCAR2 & chr12:122695781-122710104 & -1.89 & Down & $2.00 \times 10^{-04}$ \\
\hline ADM & chr11:10304979-10307402 & -1.67 & Down & $2.00 \times 10^{-04}$ \\
\hline MAK & chr6:10762722-10838954 & -2.07 & Down & $2.50 \times 10^{-04}$ \\
\hline ABCG1 & chr21:42199688-42304389 & -1.98 & Down & $4.50 \times 10^{-04}$ \\
\hline LGALSL & chr2:64454192-64461383 & -1.69 & Down & $5.00 \times 10^{-04}$ \\
\hline BTNL8 & chr5:180899076-180952166 & -1.89 & Down & $5.50 \times 10^{-04}$ \\
\hline CCNJL & chr5:160251651-160339592 & -1.84 & Down & $6.50 \times 10^{-04}$ \\
\hline HIP1 & chr7:75533297-75738976 & -1.51 & Down & $6.50 \times 10^{-04}$ \\
\hline SOCS3 & chr17:78356776-78360079 & -1.85 & Down & $8.00 \times 10^{-04}$ \\
\hline PADI2 & chr1:17066760-17119453 & -1.85 & Down & $8.00 \times 10^{-04}$ \\
\hline TGFA & chr2:70402845-70554015 & -1.60 & Down & $1.05 \times 10^{-03}$ \\
\hline KIAA0226L & chr13:46341999-46390042 & -1.35 & Down & $1.30 \times 10^{-03}$ \\
\hline OSM & chr22:30262827-30266843 & -1.65 & Down & $1.40 \times 10^{-03}$ \\
\hline CRISPLD2 & chr16:84819980-84909510 & -1.44 & Down & $1.55 \times 10^{-03}$ \\
\hline KCNS1 & chr20:45091213-45101112 & -1.37 & Down & $1.55 \times 10^{-03}$ \\
\hline IL1B & chr2:112829757-112836842 & -1.52 & Down & $1.70 \times 10^{-03}$ \\
\hline KAZN & chr1:13893386-15220480 & -2.41 & Down & $2.10 \times 10^{-03}$ \\
\hline HLX & chr1:220832762-220885059 & -1.31 & Down & $2.20 \times 10^{-03}$ \\
\hline ABCA1 & chr9:104781001-104939096 & -2.05 & Down & $2.25 \times 10^{-03}$ \\
\hline HSPA1A & chr6:31815513-31817942 & -1.45 & Down & $2.35 \times 10^{-03}$ \\
\hline KREMEN1 & chr22:29058671-29168333 & -1.52 & Down & $2.45 \times 10^{-03}$ \\
\hline
\end{tabular}

DEG, differentially expressed genes.

LOC101928143 (degree=154) and LINC00963 (degree=154). The negatively co-expressed DElncRNA-DEmRNA network (Fig. 2B) consisted of 175 nodes and 301 edges, and its hub lncRNAs were LINC01094 (degree=135) and LOC105371455 (degree $=85)$.

Nearby DEmRNAs of DElncRNAs. A total of 97 DElncRNAs nearby DEmRNA pairs were obtained. LOC101928595, LOC101929866 and HCG27 had 13, 8 and 7 nearby DEmRNAs, respectively, and were the top three DElncRNAs with the most nearby DEmRNAs (Table IV). DElncRNAs nearby DEmRNA pairs in which the expression levels of DEmRNAs were co-expressed with DElncRNAs are listed in Table V.

Functional annotation. Based on the GO enrichment analysis of DEmRNAs co-expressed with DElncRNAs, inflammatory response $(\mathrm{FDR}=3.14 \mathrm{E}-5)$, anti-apoptosis $(\mathrm{FDR}=5.44 \mathrm{E}-05)$, plasma membrane $(\mathrm{FDR}=1.48 \mathrm{E}-11)$, integral to membrane
$(\mathrm{FDR}=6.06 \mathrm{E}-10)$, protein binding $(\mathrm{FDR}=1.39 \mathrm{E}-06)$, and receptor activity $(\mathrm{FDR}=8.92 \mathrm{E}-06)$ were the most significantly enriched GO terms in PMOP (Fig. 3A-C). Hematopoietic cell lineage $(\mathrm{FDR}=0.000244565)$, Osteoclast differentiation $(\mathrm{FDR}=0.000438367)$ and Cytokine-cytokine receptor interaction (FDR=0.00212347) were the most significantly enriched KEGG pathways in PMOP (Fig. 3D).

Validation in the GEO dataset. The expression patterns of selected DElncRNAs (LINC00963, LOC105376834, LOC101929866, LOC105374771 and LOC100506113) and DEmRNAs [alkaline phosphatase, liver/bone/kidney (ALPL), suppressor of cytokine signaling 3 (SOCS3), secretory leukocyte peptidase inhibitor (SLPI) and CD177] were verified using the GSE56815 dataset. As shown in Fig. 4A-D, SOCS3, SLPI and CD177 were upregulated in PMOP, which was consistent with the RNA-sequencing results. ALPL was downregulated in PMOP, which was inconsistent with the RNA-sequencing results. However, only one of these five 
Table III. Significantly differentially expressed lncRNAs in patients with postmenopausal osteoporosis compared with normal controls.

\begin{tabular}{|c|c|c|c|c|}
\hline LncRNA & Locus & Regulation & $\log 2$ (fold change) & P-value \\
\hline LOC105374771 & chr2:64390955-64425399 & Down & -2.57 & $5.00 \times 10^{-05}$ \\
\hline LOC105372321 & chr19:21444103-21464331 & Up & 3.58 & $5.00 \times 10^{-05}$ \\
\hline PSMD5-AS1 & chr9:120843041-120854373 & Down & -1.70 & $1.00 \times 10^{-04}$ \\
\hline PAX8-AS1 & chr2:113215996-113278950 & Down & -2.41 & $7.00 \times 10^{-04}$ \\
\hline LOC105372578 & chr20:24919978-24932985 & Down & -2.70 & $1.65 \times 10^{-03}$ \\
\hline LINC00570 & chr2:11393980-11403077 & Up & 2.20 & $1.95 \times 10^{-03}$ \\
\hline LOC105369213 & chr16:81739026-81777351 & Down & -1.82 & $5.00 \times 10^{-05}$ \\
\hline LOC105378020 & chr6:137943074-137957648 & Up & $\inf$ & $3.75 \times 10^{-03}$ \\
\hline SNHG5 & chr6:85677006-85678733 & Up & 1.83 & $4.15 \times 10^{-03}$ \\
\hline LOC105378415 & chr10:88061829-88104391 & Down & -1.37 & $4.80 \times 10^{-03}$ \\
\hline LOC105374150 & chr3:148439991-148465791 & Up & 1.76 & 0.01 \\
\hline LINC00282 & chr13:51804681-51845150 & Down & -1.69 & 0.01 \\
\hline LOC102724231 & chr3:44421131-44424025 & Down & -1.46 & 0.01 \\
\hline LINC00211 & chr2:37826246-37875863 & Down & -1.92 & 0.01 \\
\hline LOC101929638 & chr22:29180622-29205834 & Up & 1.77 & 0.01 \\
\hline JHDM1D-AS1 & chr7:140177260-140179640 & Up & 1.77 & 0.01 \\
\hline LOC105370449 & chr14:34551436-34557529 & Up & 2.89 & 0.01 \\
\hline LOC105372881 & chr1:207365821-207373252 & Down & -1.14 & 0.01 \\
\hline LOC 105373262 & chr1:244230505-244325182 & Down & -1.75 & 0.01 \\
\hline LOC105371455 & chr1:157225405-157283617 & Up & 1.68 & 0.01 \\
\hline LOC100507487 & chr4:128428015-128519398 & Up & 3.25 & 0.01 \\
\hline LOC101929866 & chr20:45178476-45191638 & Down & -1.37 & 0.01 \\
\hline LINC00963 & chr9:129488659-129513686 & Down & -1.08 & 0.02 \\
\hline LOC101928143 & chr14:73460934-73463642 & Down & -1.16 & 0.02 \\
\hline LOC399715 & chr10:6326543-6335982 & Down & -1.37 & 0.02 \\
\hline LOC105373730 & chr2:165821976-165848198 & Up & 1.43 & 0.02 \\
\hline LOC100507639 & chr4:141321123-141332617 & Up & 1.45 & 0.02 \\
\hline LOC 105374768 & chr2:64299870-64344064 & Down & -1.34 & 0.02 \\
\hline LOC100506159 & chr12:9936578-9943495 & Down & -1.91 & 0.02 \\
\hline LOC105376834 & chr1:21585689-21591187 & Down & -2.23 & 0.02 \\
\hline LINC01094 & chr4:78645993-78684501 & Up & 1.29 & 0.02 \\
\hline LOC105378085 & chr6:159586906-159604657 & Down & -1.36 & 0.02 \\
\hline LOC105377067 & chr3:46130889-46190381 & Down & -1.26 & 0.02 \\
\hline LOC105369823 & chr12:69624414-69699416 & Up & 2.14 & 0.02 \\
\hline LOC101929422 & chr14:101120762-101123545 & Up & 2.50 & 0.02 \\
\hline LOC105375328 & chr7:64944845-64950665 & Up & 1.73 & 0.02 \\
\hline LINC01271 & chr20:50292719-50321342 & Down & -2.13 & 0.03 \\
\hline LOC102723828 & chr4:31997378-32155406 & Up & inf & 0.03 \\
\hline LOC105377384 & chr4:116344095-116355205 & Up & 2.43 & 0.03 \\
\hline LOC100506113 & chr11:75801640-75814797 & Down & -1.26 & 0.03 \\
\hline LOC105377782 & chr8:2199669-2206204 & Up & 1.20 & 0.03 \\
\hline HCG27 & chr6:31197759-31203968 & Down & -0.90 & 0.03 \\
\hline LINC01137 & chr1:37454878-37474443 & Down & -1.84 & 0.03 \\
\hline LOC105374546 & chr4:26859623-26860599 & Up & 3.84 & 0.03 \\
\hline LOC105376995 & chr20:62533992-62536728 & Up & 1.78 & 0.03 \\
\hline LOC105374852 & chr2:88016353-88021354 & Up & 1.72 & 0.04 \\
\hline LOC105378701 & chr1:47172216-47177080 & Up & 2.11 & 0.04 \\
\hline LOC101928595 & chr16:30096429-30113557 & Down & -0.98 & 0.04 \\
\hline LOC105372991 & chr22:30447958-30472047 & Up & 1.33 & 0.04 \\
\hline LOC105374769 & chr2:64299870-64344064 & Down & -3.63 & 0.05 \\
\hline GAS5 & chr1:173863247-173867987 & Up & 1.03 & 0.05 \\
\hline
\end{tabular}


Table IV. Nearby DEmRNAs of DElncRNAs in postmenopausal osteoporosis.

\begin{tabular}{|c|c|c|c|c|}
\hline Count & DElncRNA & lncRNA location & mRNA & mRNA location \\
\hline 1 & LOC105376834 & chr1:21585689-21591187 & ALPL & $\operatorname{chr} 1: 21508981-21578412$ \\
\hline \multirow[t]{4}{*}{4} & LINC01137 & chr1:37454878-37474443 & $\mathrm{ZC} 3 \mathrm{H} 12 \mathrm{~A}$ & chr1:37474517-37484377 \\
\hline & & & SNIP1 & chr1:37531436-37554344 \\
\hline & & & DNALI1 & chr1:37556918-37595985 \\
\hline & & & GNL2 & chr1:37556918-37595985 \\
\hline \multirow[t]{2}{*}{2} & LOC105378701 & chr1:47172216-47177080 & CYP4Z1 & chr1:47067487-47118320 \\
\hline & & & CYP4A22 & chr1:47137424-47149738 \\
\hline 1 & LOC105371455 & $\operatorname{chr} 1: 157225405-157283617$ & ETV3 & chr1:157121190-157138591 \\
\hline \multirow[t]{4}{*}{4} & GAS5 & chr1:173863247-173867987 & CENPL & chr1:173799549-173824639 \\
\hline & & & ZBTB37 & chr1:173868094-173891122 \\
\hline & & & SERPINC1 & chr1:173903803-173917378 \\
\hline & & & $\mathrm{RC} 3 \mathrm{H} 1$ & chr1:173931083-173993072 \\
\hline \multirow[t]{2}{*}{2} & LOC105372881 & chr1:207365821-207373252 & CD55 & chr1:207321471-207360966 \\
\hline & & & $\mathrm{CR} 2$ & chr1:207454299-207489895 \\
\hline 1 & LOC105373262 & chr1:244230505-244325182 & $\mathrm{C} 1$ orf100 & chr1:244352062-244389896 \\
\hline 1 & LINC00570 & chr2:11393980-11403077 & E2F6 & chr2:11444374-11466177 \\
\hline 1 & LOC105374771 & chr2:64390955-64425399 & LGALSL & chr2:64454192-64461383 \\
\hline \multirow[t]{2}{*}{2} & LOC105374852 & chr2:88016353-88021354 & RGPD2 & chr2:87748086-87992864 \\
\hline & & & SMYD1 & chr2:88067779-88113384 \\
\hline 1 & LOC105373730 & chr2:165821976-165848198 & GALNT3 & chr2:165747802-165796352 \\
\hline 1 & LOC102724231 & & TOPAZ1 & chr3:44241885-44338010 \\
\hline \multirow[t]{2}{*}{2} & LOC105377067 & chr3:46130889-46190381 & XCR1 & chr3:46016989-46086803 \\
\hline & & & CCR1 & chr3:46201708-46208341 \\
\hline 1 & LOC105374546 & chr4:26859623-26860599 & STIM2 & chr4:26860690-27025381 \\
\hline 1 & LINC01094 & chr4:78645993-78684501 & ANXA3 & chr4:78551587-78610451 \\
\hline 1 & LOC100507639 & chr4:141321123-141332617 & ZNF330 & chr4:141220293-141234697 \\
\hline \multirow[t]{7}{*}{7} & HCG27 & chr6:31197759-31203968 & C6orf15 & chr6:31111222-31112555 \\
\hline & & & PSORS1C1 & chr6:31114830-31140092 \\
\hline & & & CDSN & chr6:31114830-31140092 \\
\hline & & & PSORS1C2 & chr6:31114830-31140092 \\
\hline & & & CCHCR1 & chr6:31142438-31158238 \\
\hline & & & POU5F1 & chr6:31164336-31170693 \\
\hline & & & HLA-C & chr6:31268748-31272136 \\
\hline 1 & LOC105378020 & chr6:137943074-137957648 & TNFAIP3 & chr6:137823668-137883314 \\
\hline 1 & LOC105378085 & chr6:159586906-159604657 & SOD2 & chr6:159679063-159789703 \\
\hline \multirow[t]{4}{*}{4} & LOC105375328 & chr7:64944845-64950665 & ZNF138 & chr7:64794387-64853800 \\
\hline & & & LOC441239 & chr7:64882492-64937316 \\
\hline & & & ZNF117 & chr7:64974451-65006746 \\
\hline & & & ERV3-1 & chr7:64974451-65006746 \\
\hline \multirow[t]{2}{*}{2} & LINC00963 & chr9:129488659-129513686 & NTMT1 & chr9:129608883-129642169 \\
\hline & & & C9orf50 & chr9:129608883-129642169 \\
\hline \multirow[t]{2}{*}{2} & LOC399715 & chr10:6326543-6335982 & PFKFB3 & chr10:6144801-6254648 \\
\hline & & & PRKCQ & chr10:6393037-6585361 \\
\hline \multirow[t]{2}{*}{2} & LOC105378415 & chr10:88061829-88104391 & PTEN & chr10:87863437-87975287 \\
\hline & & & RNLS & chr10:88131897-88583860 \\
\hline \multirow[t]{2}{*}{2} & LOC100506113 & chr11:75801640-75814797 & MOGAT2 & chr11:75701595-75732958 \\
\hline & & & DGAT2 & chr11:75768732-75801536 \\
\hline \multirow[t]{4}{*}{4} & LOC100506159 & chr12:9936578-9943495 & KLRF2 & chr12:9881488-9932430 \\
\hline & & & CLEC2A & chr12:9881488-9932430 \\
\hline & & & CLEC12B & chr12:10006137-10030606 \\
\hline & & & CLEC9A & chr12:10030676-10066030 \\
\hline \multirow[t]{2}{*}{2} & LOC105369823 & chr12:69624414-69699416 & LRRC10 & chr12:69608563-69611162 \\
\hline & & & BEST3 & chr12:69624414-69699416 \\
\hline
\end{tabular}


Table IV. Continued.

\begin{tabular}{|c|c|c|c|c|}
\hline Count & DElncRNA & lncRNA location & mRNA & mRNA location \\
\hline \multirow[t]{4}{*}{4} & \multirow[t]{4}{*}{ LINC00282 } & \multirow[t]{4}{*}{ chr13:51804681-51845150 } & WDFY2 & chr13:51584193-51804206 \\
\hline & & & DHRS12 & chr13:51584193-51804206 \\
\hline & & & CCDC70 & chr13:51861980-51866236 \\
\hline & & & ATP7B & chr13:51932668-52012130 \\
\hline \multirow[t]{3}{*}{3} & \multirow[t]{3}{*}{ LOC101928143 } & \multirow[t]{3}{*}{ chr14:73460934-73463642 } & NUMB & chr14:73275209-73458580 \\
\hline & & & HEATR4 & chr14:73478483-73634418 \\
\hline & & & C14orf169 & chr14:73478483-73634418 \\
\hline \multirow[t]{13}{*}{13} & \multirow[t]{13}{*}{ LOC101928595 } & \multirow[t]{13}{*}{ chr16:30096429-30113557 } & INO80E & chr16:29996208-30023280 \\
\hline & & & DOC2A & chr16:29996208-30023280 \\
\hline & & & C16orf92 & chr16:30023333-30053026 \\
\hline & & & FAM57B & chr16:30023333-30053026 \\
\hline & & & ALDOA & $\operatorname{chr} 16: 30053089-30070420$ \\
\hline & & & PPP4C & chr16:30075975-30085377 \\
\hline & & & TBX6 & chr16:30085792-30091919 \\
\hline & & & GDPD3 & chr16:30096429-30113557 \\
\hline & & & MAPK3 & chr16:30114104-30123309 \\
\hline & & & CORO1A & chr16:30183392-30189076 \\
\hline & & & BOLA2B & chr16:30192929-30206927 \\
\hline & & & SLX1A & chr16:30192929-30206927 \\
\hline & & & SULT1A3 & chr16:30192929-30206927 \\
\hline \multirow[t]{2}{*}{2} & \multirow[t]{2}{*}{ LOC105369213 } & \multirow[t]{2}{*}{ chr16:81739026-81777351 } & CMIP & chr16:81445169-81711762 \\
\hline & & & PLCG2 & chr16:81779257-81962693 \\
\hline \multirow[t]{3}{*}{3} & \multirow[t]{3}{*}{ LOC105372578 } & \multirow[t]{3}{*}{ chr20:24919978-24932985 } & CST7 & chr20:24949229-24959928 \\
\hline & & & APMAP & chr20:24962924-24992974 \\
\hline & & & ACSS1 & chr20:25006229-25058182 \\
\hline \multirow[t]{8}{*}{8} & \multirow[t]{8}{*}{ LOC101929866 } & \multirow[t]{8}{*}{ chr20:45178476-45191638 } & STK4 & chr20:44966473-45079977 \\
\hline & & & KCNS1 & chr20:45091213-45101112 \\
\hline & & & WFDC5 & chr20:45109451-45116321 \\
\hline & & & WFDC12 & chr20:45123425-45124465 \\
\hline & & & PI3 & chr20:45174898-45176544 \\
\hline & & & SEMG1 & chr20:45206963-45209773 \\
\hline & & & SEMG2 & chr20:45221368-45224458 \\
\hline & & & SLPI & chr20:45230820-45290352 \\
\hline 1 & LINC01271 & chr20:50292719-50321342 & LINC01272 & chr20:50267467-50279795 \\
\hline \multirow[t]{2}{*}{2} & \multirow[t]{2}{*}{ LOC105376995 } & \multirow[t]{2}{*}{ chr20:62533992-62536728 } & GATA5 & chr20:62463496-62475970 \\
\hline & & & MIR1-1HG & chr20:62543069-62570764 \\
\hline \multirow[t]{3}{*}{3} & \multirow[t]{3}{*}{ LOC101929638 } & \multirow[t]{3}{*}{ chr22:29180622-29205834 } & KREMEN1 & chr22:29058671-29168333 \\
\hline & & & RHBDD3 & chr22:29259854-29300525 \\
\hline & & & EWSR1 & chr22:29259854-29300525 \\
\hline 5 & LOC105372991 & chr22:30447958-30472047 & SF3A1 & chr22:30331987-30378655 \\
\hline & & & SEC14L2 & chr22:30396940-30436501 \\
\hline & & & SEC14L3 & chr22:30447958-30472047 \\
\hline & & & SEC14L6 & chr22:30522796-30546717 \\
\hline & & & GAL3ST1 & chr22:30554634-30574588 \\
\hline
\end{tabular}

DElncRNAs, differentially expressed long non-coding RNAs.

DElncRNAs, LINC00963, was detected in GSE56815, which may be due to the restriction of the microarray. LINC00963 was downregulated in PMO, which showed the same pattern with that in the RNA-sequencing results (Fig. 4E).
The expression pattern of six DElncRNAs (PSMD5-AS1, PAX8-AS1, JHDM1D-AS1, LINC00963, LOC100506113 and HCG27) was validated by GSE7158. Five DElncRNAs (PSMD5-AS1, PAX8-AS1, LINC00963, LOC100506113 and 


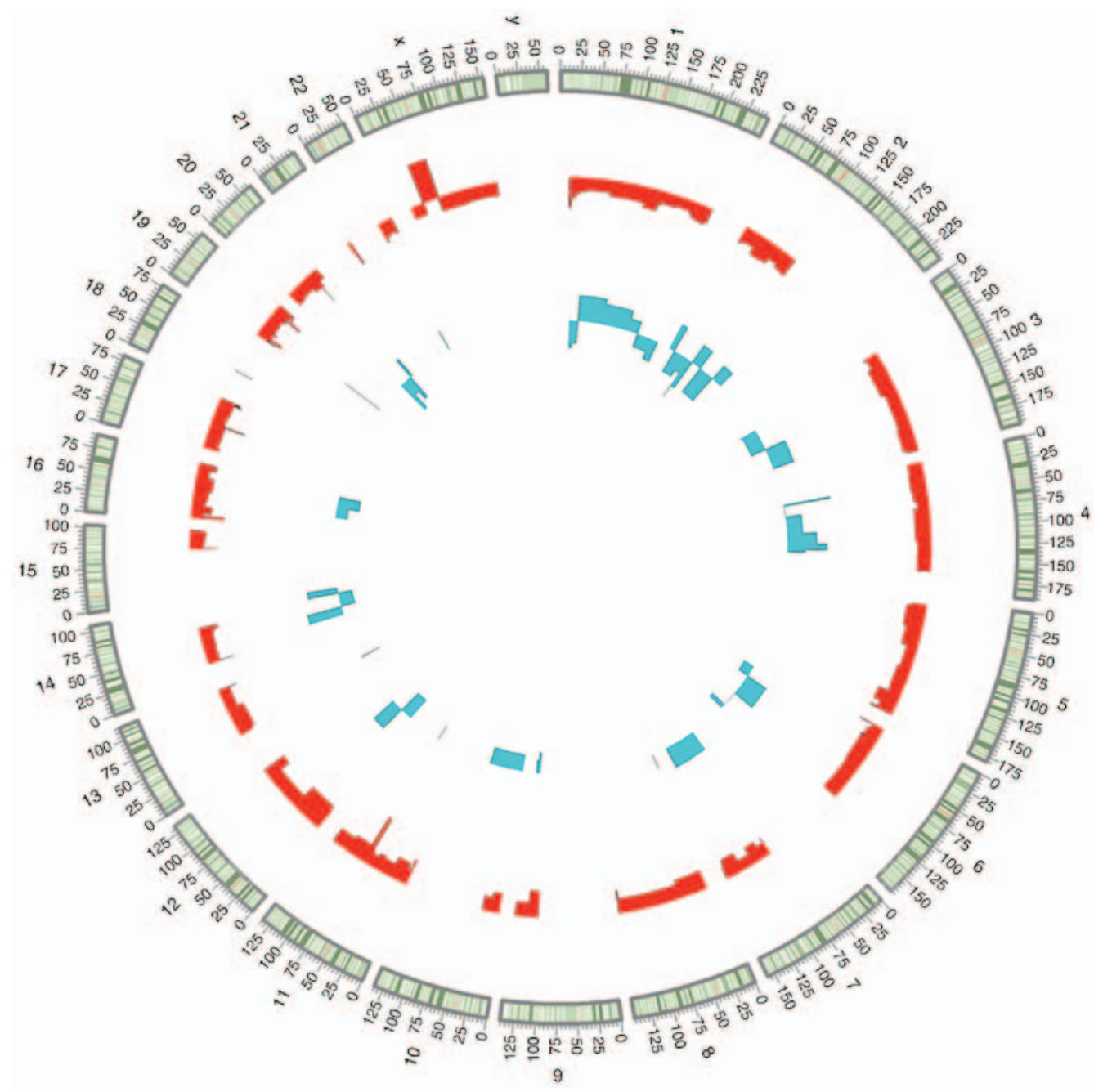

Figure 1. Circus plots of the distribution of DElncRNAs and DEmRNAs in PMOP on chromosomes. The outer layer cycle represents the chromosome map of the human genome hg19. The red inner layer and blue inner layer represent the distribution of DEmRNAs and DElncRNAs in PMOP on different chromosomes, respectively. DE, differentially expressed; lncRNAs, long non-coding RNAs; PMOP, post-menopausal osteoporosis.

Table V. DElncRNA-nearby DEmRNA pairs in which DEmRNAs are co-expressed with DElncRNAs.

\begin{tabular}{lccc}
\hline DEmRNA & DElncRNA & PCC & P-value \\
\hline DGAT2 & LOC100506113 & $9.77 \times 10^{-01}$ & $4.05 \times 10^{-03}$ \\
KCNS1 & LOC101929866 & $9.02 \times 10^{-01}$ & $3.64 \times 10^{-02}$ \\
PI3 & LOC101929866 & $9.65 \times 10^{-01}$ & $7.89 \times 10^{-03}$ \\
SLPI & LOC101929866 & $9.48 \times 10^{-01}$ & $1.41 \times 10^{-02}$ \\
LGALSL & LOC105374771 & $9.93 \times 10^{-01}$ & $6.56 \times 10^{-04}$ \\
ALPL & LOC105376834 & $9.60 \times 10^{-01}$ & $9.51 \times 10^{-03}$ \\
\hline
\end{tabular}

DE, differentially expressed; lncRNA, long non-coding RNA; PCC, Pearson's correlation coefficient; DGAT2, diacylglycerol O-acyltransferase 2; KCNS1, potassium voltage-gated channel subfamily S member 1; PI3, peptidase inhibitor 3; SLPI, secretory leukocyte peptidase inhibitor; LGALSL, galectin-related protein; ALPL, alkaline phosphatase, liver/bone/kidney.

HCG27) were downregulated, whereas JHDM1D-AS1 was upregulated, in PMOP compared with NC (Fig. 5), which was the same pattern found in the RNA-sequencing results.

\section{Discussion}

Although the function of the majority of lncRNAs remains to be elucidated, previous studies have indicated that lncRNAs may be involved in the pathogenesis of PMOP. Identifying the key DElncRNAs in PMOP not only provides novel clues for understanding the function of IncRNAs, but also contributes to developing novel biomarkers of PMOP.

In the present study, the landscape of lncRNAs in PMOP was obtained and a total of 51 DElncRNAs in PMOP were identified. With the exception of LINC00963 and GAS5, no previous study has reported on the function of the remaining 49 DElncRNAs. In addition, the present study is the first, to the best of our knowledge, to show that these 51 DElncRNAs may be associated with PMOP.

LINC00963 is reported to be involved in cell viability, motility and invasiveness in prostate cancer cells by affecting the expression of epidermal growth factor receptor (18). In the present study, LINC00963 was a significantly downregulated lncRNA in PMOP. Whether LINC00963 is involved in PMOP by regulating the viability, motility and invasiveness of osteoclasts and osteoblast requires further investigation.

Although the functions of lncRNAs remain to be fully elucidated, previous studies have indicated that IncRNAs are 

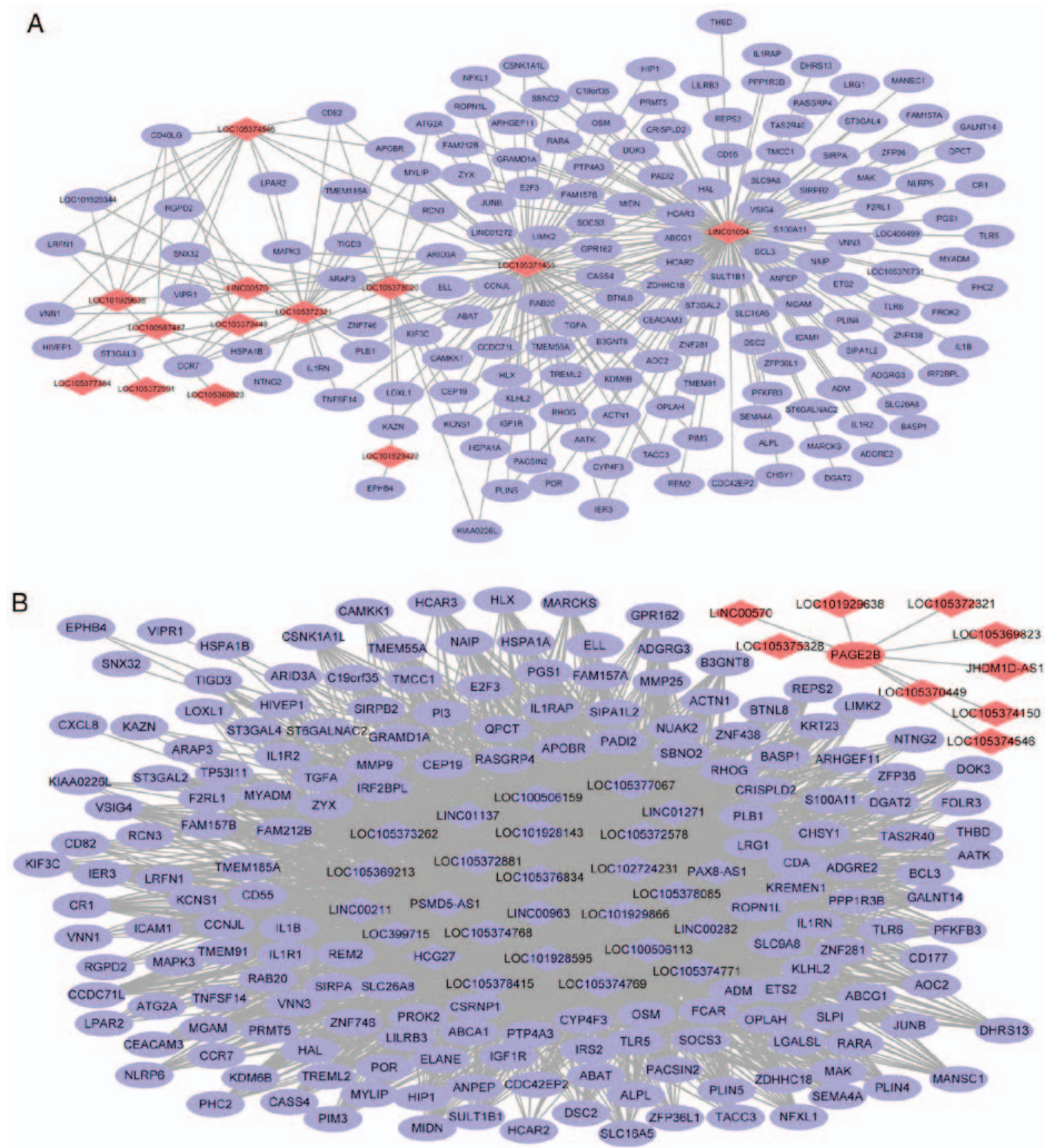

Figure 2. DElncRNA-DEmRNA co-expression network. The PCC between the expression levels of each DElncRNA-DEmRNA pair in PMOP and NC groups were calculated. (A) DElncRNA-DEmRNA pairs with PCC $\geq 0.90$ and $\mathrm{P}<0.05$ were considered to be positively co-expressed DElncRNA-DEmRNA pairs and (B) DElncRNA-DEmRNA pairs with PCC $\leq-0.90$ and $\mathrm{P}<0.05$ were considered to be negatively co-expressed DElncRNA-DEmRNA pairs. The rhombi and the ellipses represent DElncRNAs and DEmRNAs in PMOP, respectively. The blue and red colors represent downregulation and upregulation in PMOP, respectively. DE, differentially expressed; lncRNAs, long non-coding RNAs; PMOP, post-menopausal osteoporosis; PCC, Pearson's correlation coefficient; NC, normal control.

important in regulating the expression levels of genes and proteins, and are involved in a variety of biochemical processes and diseases (19-21). To date, calculating the correlation coefficients between the expression levels of lncRNAs and genes has been the most popular approach to identify potential target genes of lncRNAs $(22,23)$. Accumulated evidence has indicated that IncRNA-mRNA co-expression analysis can be used to examine the biological functions of lncRNAs in various diseases by examining their co-expressed mRNAs (24-26). In addition, several lncRNA-gene pairs have been validated by in vitro experiments (27).
In the present study, LINC00963 was a hub lncRNA of the positively co-expressed DElncRNA-DEmRNA network. Among its 154 co-expressed DEmRNAs, SOCS3 and adrenomedullin (ADM) were two of the top 20 DEmRNAs in PMOP. ADM is a 52-amino acid peptide with several biological functions. Previous studies have demonstrated that ADM is closely associated with regulating bone formation (28). The expression of ADM has been detected in chondrocytes and osteoblasts (29). ADM can promote growth of chondrocytes and osteoblasts in vitro (29). Additionally, apoptotic cell death in serum-starved osteoblasts can be reduced by ADM (30). 
A

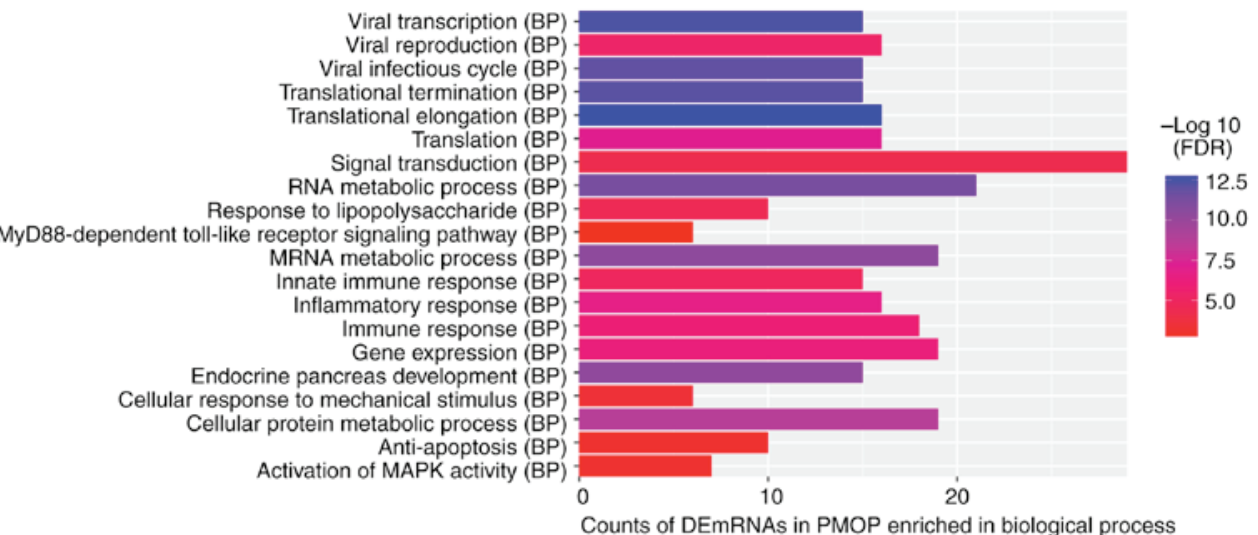

B Zinc ion binding (MF)
Transmembrane signaling receptor activity (MF)
Transferase activity, transfering acyl groups (MF)
Structural constituent of ribosome (MF)
cific DNA binding transcription factor activity (MF) Zinc ion binding (MF)
Transmembrane signaling receptor activity (MF)
Transferase activity, transferring acyl groups (MF)
Structural constituent of ribosome (MF) Sequence-specific DNA binding (MF)
Receptor activity (MF) Protein serine/threonine kinase activity (MF Protein dimerization activity (MF) Protein-arginine deiminase activity (MF Phospholipid transporter activity (MF)

Oxygen transporter activity (MF Nucleotide binding (MF Metal ion binding (MF Enzyme binding (MF)
Cytokine receptor activity (MF) Cholesterol binding (MF Arachidonate 5-ipoxygenase activity (MF

Counts of DEmRNAs in PMOP enriched in biological process

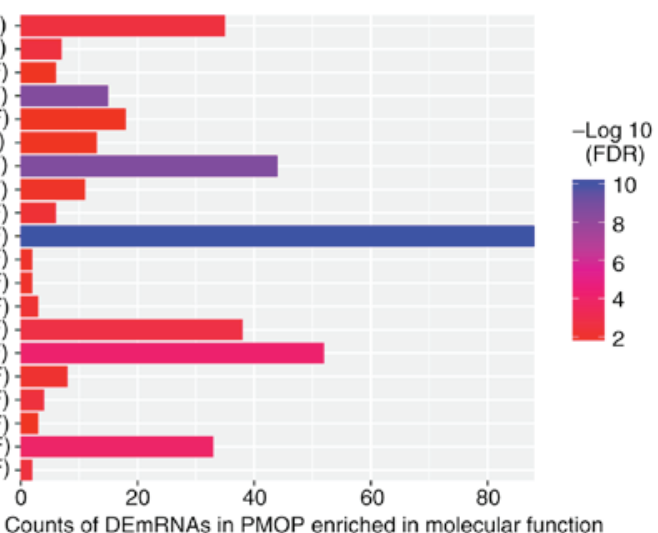

C
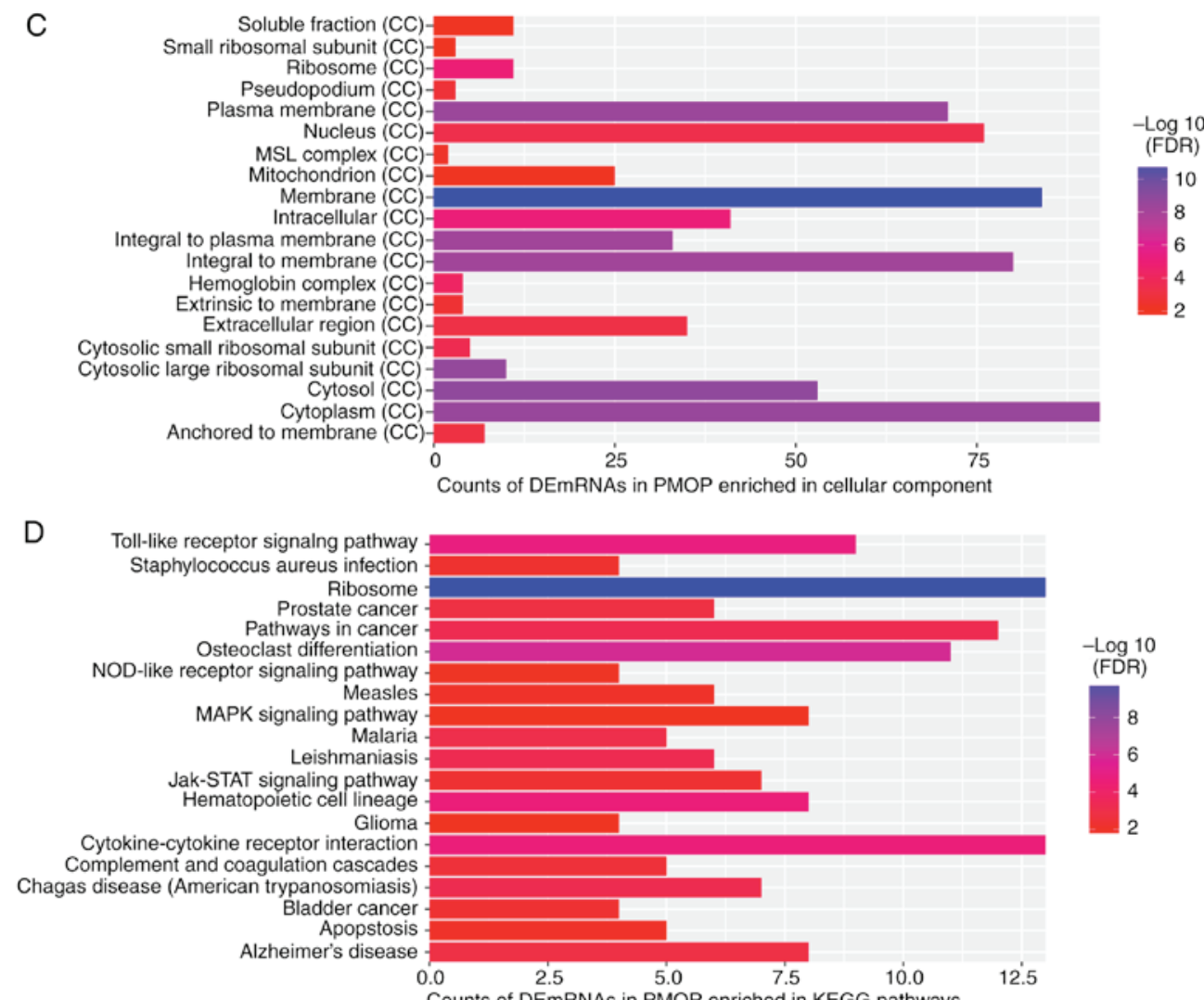

Counts of DEmRNAs in PMOP enriched in KEGG pathways

Figure 3. Significantly enriched GO terms and KEGG pathways of DEmRNAs co-expressed with DElncRNAs in PMOP. GO and KEGG pathway enrichment analyses of DEmRNAs co-expressed with DElncRNAs was performed using the online GeneCoDis3 tool (http://genecodis.cnb.csic.es/analysis). A P-value was obtained using a hypergeometric test. FDR (corrected P-value) $<0.05$ was set as the cut-off for significant GO terms and KEGG pathways. The y-axis shows GO terms or KEGG pathways and the x-axis presents counts of DEmRNAs in PMOP enriched in GO terms or KEGG pathways. The color scale represented -log FDR (A) BP; (B) MF; (C) CC; (D) KEGG pathways. GO, Gene Ontology; KEGG, Kyoto Encyclopedia of Genes and Genomes; DE, differentially expressed; lncRNAs, long non-coding RNAs; PMOP, post-menopausal osteoporosis; BP, biological process; MF, molecular function; CC, cellular component; FDR, false discovery rate. 
A

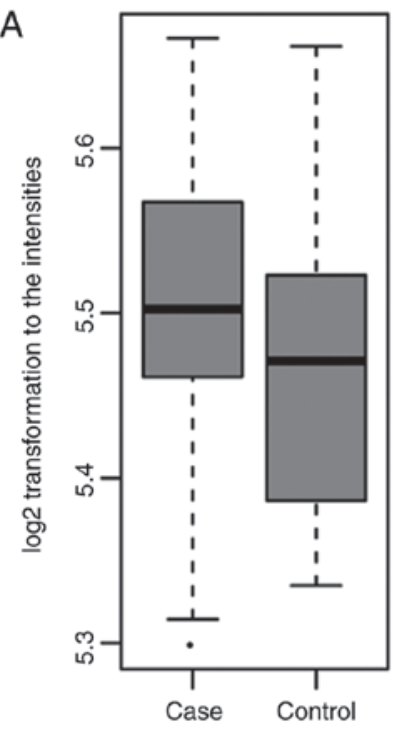

B

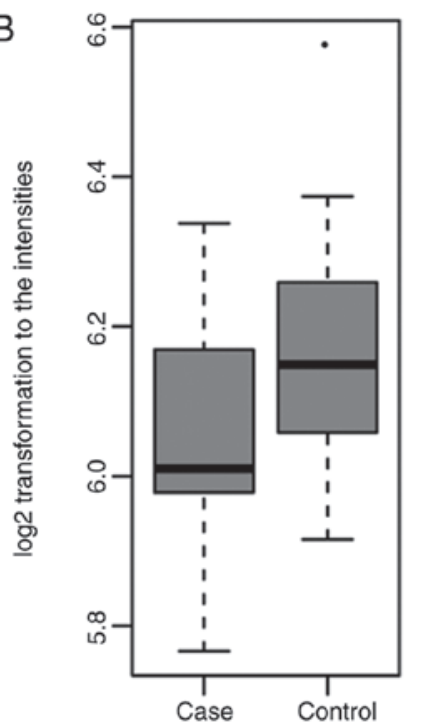

C

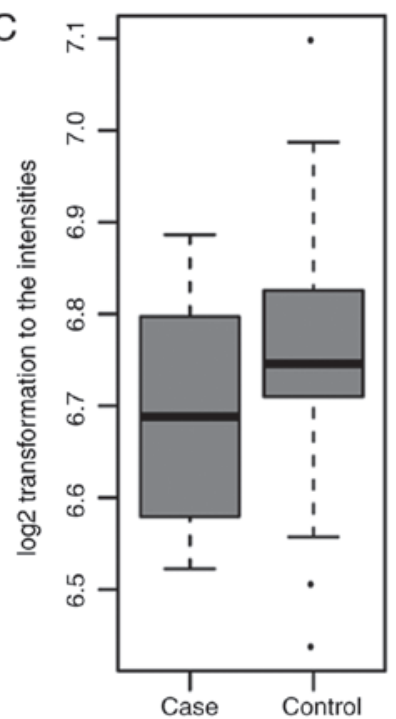

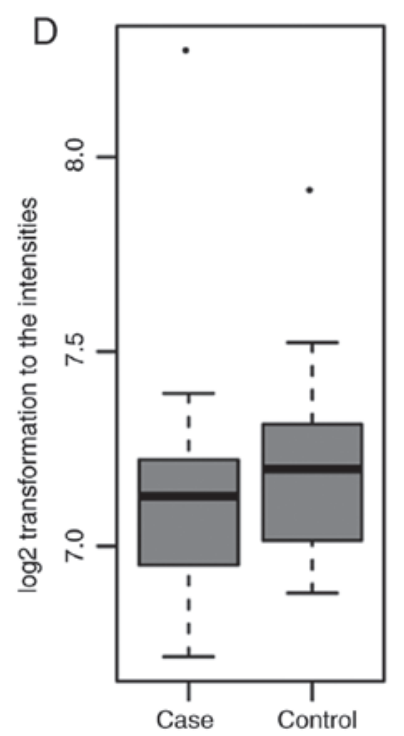

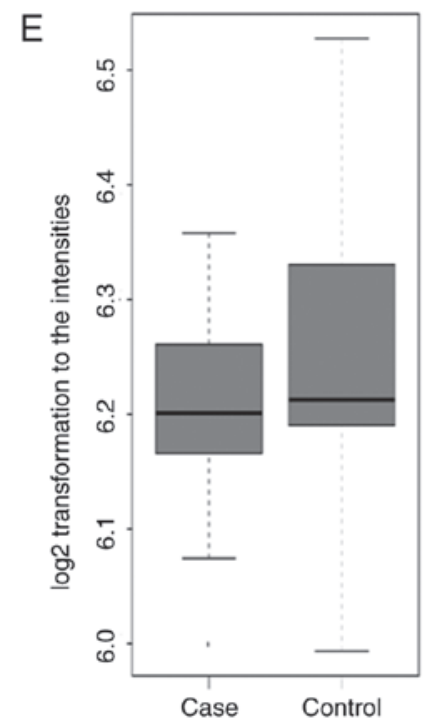

Figure 4. Validation of selected DElncRNAs and DEmRNAs in GSE56815. The x-axes show PMOP (case) and normal control groups, and the y-axes show the $\log 2$ transformation to the intensities. Student's t-test was used to calculate the significant differences in DElncRNAs and DEmRNAs between PMOP and normal control groups. (A) alkaline phosphatase, liver/bone/kidney; (B) suppressor of cytokine signaling 3; (C) CD177; (D) secretory leukocyte peptidase inhibitor; (E) LINC00963. DE, differentially expressed; lncRNAs, long non-coding RNAs; PMOP, post-menopausal osteoporosis.

In the present study, the expression of ADM was significantly downregulated in the blood samples of patients with PMOP. It was hypothesized that downregulated ADM may be involved in PMOP through reducing the bone formation induced by the reduced proliferation of osteoblasts. The SOCS family includes cytokine-inducible negative regulators of cytokine signaling. As a member of the SOCS family, SOCS3 can be regulated by various cytokines (31). Previous studies have reported that increased SOCS3 elevated transforming growth factor- $\beta, \mathrm{TNF}-\alpha$ and RANK ligand (RANKL)-induced osteoclast formation, and promoted precursors to the osteoclast lineage through the inhibition of specific anti-osteoclastic Janus kinase/signal transducer and activator of transcription signals (31). In addition, increased SOCS3 is closely associated with inflammation-induced bone loss (32). SOCS3 is also involved in RANKL-mediated dendritic cell-derived osteoclastogenesis by regulating associated cytokine signaling (32). Diabetes-associated inflammation-induced alveolar bone loss can also be regulated by SOCS3. In the present study, downregulated SOCS3 was detected in blood samples of patients with PMOP, which suggested that SOCS3 may also be a regulator of PMOP. It was hypothesized that LINC00963-ADM and LINC00963-SOCS3 interactions may be key in PMOP.

Another lncRNA, GAS5, has been reported to regulate apoptosis in prostate cancer, breast cancer, renal cell carcinoma and gastric cancer (33-36). In the present study, GAS5 was a significantly upregulated DElncRNA in POMP, which had four nearby DEmRNAs (centromere protein L, zinc finger and BTB domain containing 37, serpin family $\mathrm{C}$ member 1 , and ring finger and $\mathrm{CCCH}$-type domains 1). It was hypothesized that GAS5 may be involved in PMOP by regulating apoptosis and these four genes.

LncRNAs have also been shown to regulate gene expression in cis. LOC105376834-ALPL and LOC101929866-SLPI were two DElncRNA-DEmRNA co-expression pairs in PMOP. In addition, ALPL and SLPI were nearby DEmRNAs of LOC105376834 and LOC101929866, respectively. It was 

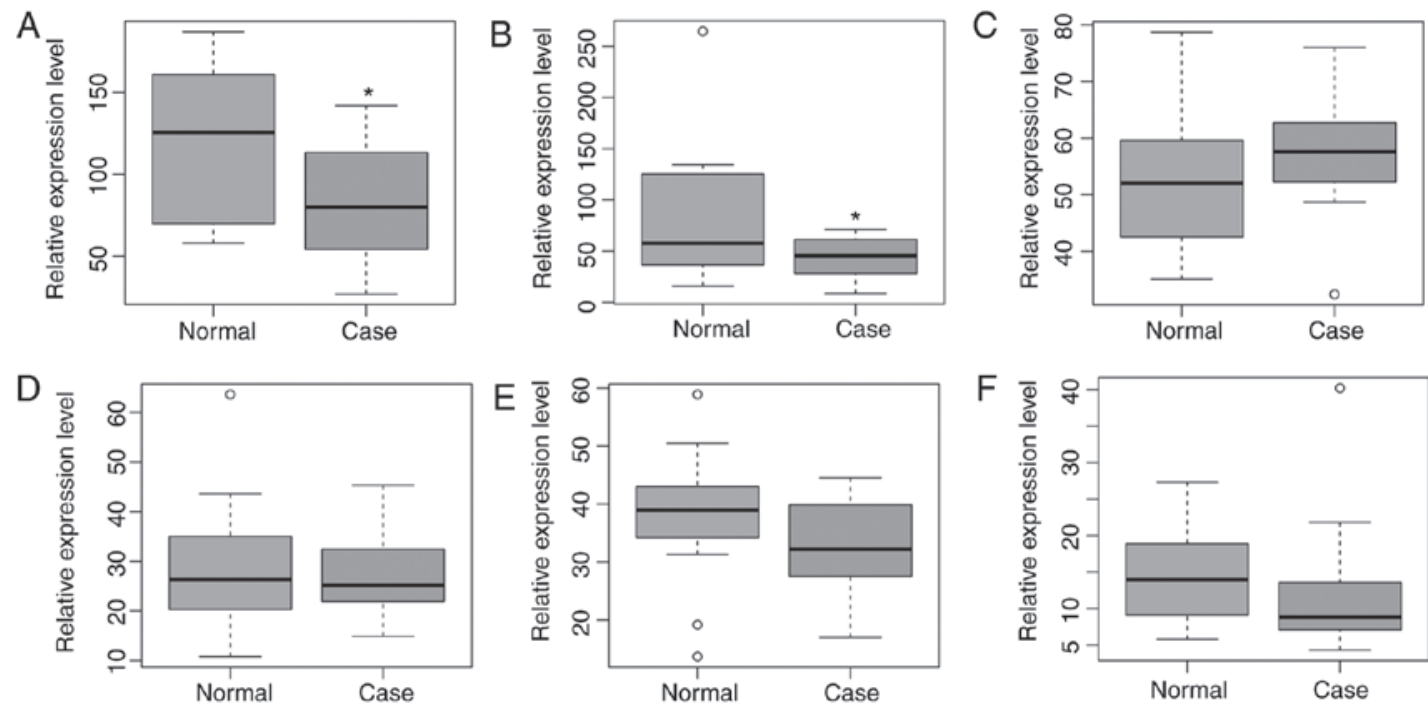

Figure 5. Validation of selected DElncRNAs in GSE7158. The x-axes show PMOP (case) and normal control groups, and the y-axes show relative expression levels. Student's t-test was used to calculate the significant differences in DElncRNAs between the PMOP and normal control groups. (A) PSMD5-AS1; (B) PAX8-AS1; (C) JHDM1D-AS1; (D) LINC00963; (E) LOC100506113; (F) HCG27. "P<0.05. DE, differentially expressed; lncRNAs, long non-coding RNAs; PMOP, post-menopausal osteoporosis.

hypothesized that LOC105376834 and LOC101929866 may regulate the expression of ALPL and SLPI by a cis-effect, in which the DElncRNAs were also co-expressed with DElncRNAs. ALPL is an osteoblast marker and is reported to be closely associated with the development of osteoporosis (37,38). Downregulated ALPL can reflect decreased activity of osteoblasts, bone formation and extracellular matrix mineralization (37). Previous studies have detected downregulated ALPL in bone tissue samples of patients with PMOP and ovariectomized mice, a model of postmenopausal osteoporosis $(37,39,40)$. In the present study, the downregulation of ALPL was detected in blood samples from patients with PMOP, which confirmed the importance of ALPL in PMOP and may serve as a diagnostic marker of PMOP. As ALPL was a nearby co-expressed DEmRNA of LOC105376834, it was hypothesized that LOC105376834 may be involved in PMOP by cis-regulating the expression of ALPL.

SLPI encodes a serine protease inhibitor, which protects epithelial tissues from serine proteases. Additionally, SLPI is an anti-inflammatory mediator (41). SLPI can contribute to wound healing by decreasing the excessive inflammatory response, elevating keratinocyte proliferation and increasing collagen deposition by suppressing the activity of protease (42). To the best of our knowledge, the association between SLPI and PMOP has not been reported previously. A significant downregulation of SLPI was detected in patients with PMOP in the present study. As accumulated evidence has indicated that various inflammatory conditions are involved with osteoporosis (43), the present study hypothesized that SLPI may be involved in PMOP by regulating the inflammatory condition. In addition, estrogen treatment has been shown to increase the expression of SPLI in alveolar epithelial cells in ovariectomized mice (44). The same result was found in the rat uterus following treatment with estrogen (45). It was hypothesized that reduced estrogen may be involved in PMOP by regulating SLPI. SLPI was the nearby co-expressed DEmRNA of LOC101929866, which suggested that LOC101929866 may be associated with PMOP. The other two nearby co-expressed DEmRNAs (potassium voltage-gated channel subfamily $\mathrm{S}$ member 1, and peptidase inhibitor 3) of LOC101929866 may also be involved in PMOP.

CD177 was the third significant DEmRNA in PMOP, which may also be an estrogen-associated gene. CD177 encodes a glycosyl-phosphatidylinositol-linked cell surface glycoprotein associated with neutrophil activation. Although there was no previous report on the association between CD177 and PMOP, a low expression of CD177 was found to be involved in clonal myeloid disorders, particularly myelodysplasia (46). A significantly upregulated level CD177 was previously detected in breast cancer cells following treatment with estrogen receptors- $\beta$ agonists (47), which suggested that CD177 was closely associated with estrogen. It was hypothesized that reduced CD177 may also be involved in PMOP by regulating estrogen. The precise role of CD177 in PMOP requires further investigation.

Besides LOC105376834 and LOC10192986, LOC105374771 and LOC100506113 were two downregulated DElncRNAs in PMOP, which had nearby co-expressed DEmRNAs. Therefore, LOC100506113 and LOC105374771 may be involved in PMOP by regulating the expression of diacylglycerol O-acyltransferase 2 and LGALSL, respectively. In addition, LOC105374771 was the most markedly downregulated lncRNA, which was co-expressed with 130 DEmRNAs, including ALPL, SOCS3, ADM, CD177 and SLPI. LOC105374771 may affect the pathogenesis of PMOP by regulating the expression of these DEmRNAs.

Besides LINC00963, the other hub IncRNAs of the positively and negatively co-expressed DElncRNAs-DEmRNAs network were LOC105378415, LOC105377067, HCG27, LOC101928143 and LINC01094. Three PMOP-associated DEmRNAs, including ALPL, SOCS3 and ADM, were common co-expressed DEmRNAs of these hub DElncRNAs, which indicated the importance of these DElncRNAs in PMOP. 
As hematopoietic cell lineage and osteoclast differentiation are two well-known pathways in PMOP. DEmRNAs enriched in these two pathways and their co-expressed DElncRNAs may be involved in PMOP by regulating hematopoietic cell lineage or osteoclast differentiation.

In conclusion, the present study identified five DEmRNAs (ALPL, SOCS3, ADM, SLPI and CD177) co-expressed with DElncRNAs, which may be involved in PMOP. DElncRNAs in PMOP, including LINC00963, LOC105376834, LOC101929866, LOC105374771 and LOC100506113, may be involved in the pathogenesis of PMOP by regulating the expression of their nearby and co-expressed DEmRNAs and the pathway of osteoclast differentiation. The results of the present study may provide a foundation for future investigations of lncRNAs in PMOP and contribute in developing novel diagnostic biomarkers and drug design for PMOP. However, the sample size for RNA sequencing in the present study was small, and the difference in body mass index between the PMOP and $\mathrm{NC}$ groups may have affected the results of RNA-sequencing, which were limitations of the study. Although the validation based on GSE56815 and GSE7158 suggested that the RNA-sequencing results were generally reliable, investigations with a larger sample size are required to confirm this conclusion. In addition, further experiments are required to address the biological significance of key lncRNAs and genes in PMOP.

\section{Acknowledgements}

Not applicable.

\section{Funding}

This study was supported by the Beijing Health System High Level Health Technical Personnel Training Project (grant no. 2015-3-009).

\section{Availability of data and materials}

The datasets used and/or analyzed during the current study are available from the corresponding author on reasonable request.

\section{Authors' contributions}

QF and AG were responsible for conceptionand design of the experiments. QF and XDB performed the experiments. XDB and JSL analyzed the data. HM and YY supplied reagents, materials and analysis tools. All named authors wrote this manuscript and have agreed to the publication of this manuscript, and it does not infringe on any copyright or property rights.

\section{Ethics approval and consent to participate}

All individuals provided written informed consent for use of their samples in the present study. The present study was approved by the Ethics Committee of Beijing Friendship Hospital, Capital Medical University (Beijing, China; 2017-P2-084-01).

\section{Consent for publication}

Not applicable.

\section{Competing interests}

The authors declare that they have no competing interests.

\section{References}

1. Zhang Y, Liu H, Zhang C, Zhang T, Zhang B, Li L, Chen G, $\mathrm{Fu} \mathrm{D}$ and Wang K: Endochondral ossification pathway genes and postmenopausal osteoporosis: Association and specific allele related serum bone sialoprotein levels in Han Chinese. Sci Rep 5: $16783,2015$.

2. Ma M, Chen X, Lu L, Yuan F, Zeng W, Luo S, Yin F and Cai J: Identification of crucial genes related to postmenopausal osteoporosis using gene expression profiling. Aging Clin Exp Res 28: 1067-1074, 2016.

3. Taguchi A, Ohtsuka M, Nakamoto T, Naito K, Tsuda M, Kudo Y, Motoyama E, Suei Y and Tanimoto K: Identification of post-menopausal women at risk of osteoporosis by trained general dental practitioners using panoramic radiographs. Dentomaxillofac Radiol 36: 149-154, 2007.

4. Sasser AC, Taylor M, Birnbaum HG, Schoenfeld MJ, Oster EF and Rousculp M: Assessing the economic impact of chronic conditions in postmenopausal women. Expert Opin Pharmacother 6: 1803-1814, 2005.

5. Sasser AC, Rousculp MD, Birnbaum HG, Oster EF, Lufkin E and Mallet D: Economic burden of osteoporosis, breast cancer, and cardiovascular disease among postmenopausal women in an employed population. Womens Health Issues 15: 97-108, 2005.

6. Richards JB, Zheng HF and Spector TD: Genetics of osteoporosis from genome-wide association studies: Advances and challenges. Nat Rev Genet 13: 576-588, 2012.

7. Liu YJ, Zhang L, Papasian CJ and Deng HW: Genome-wide association studies for osteoporosis: A 2013 update. J Bone Metab 21: 99-116, 2014.

8. Kotrych D, Dziedziejko V, Safranow K, Sroczynski T, Staniszewska M, Juzyszyn Z and Pawlik A: TNF- $\alpha$ and IL10 gene polymorphisms in women with postmenopausal osteoporosis. Eur J Obstet Gynecol Reprod Biol 199: 92-95, 2016.

9. Shang DP, Lian HY, Fu DP, Wu J, Hou SS and Lu JM: Relationship between estrogen receptor 1 gene polymorphisms and postmenopausal osteoporosis of the spine in Chinese women. Genet Mol Res 15, 2016.

10. Zhang C, Ma J, Chen G, Fu D, Li L and Li M: Evaluation of common variants in CNR2 gene for bone mineral density and osteoporosis susceptibility in postmenopausal women of Han Chinese. Osteoporos Int 26: 2803-2810, 2015.

11. Luo D, Liu Y, Zhou Y, Chen Z, Yang L, Liu Y, Xu Q, Xu H, Kuang H, Huang Q, et al: Association between dietary phytoestrogen intake and bone mineral density varied with estrogen receptor alpha gene polymorphisms in southern Chinese postmenopausal women. Food Funct 6: 1977-1983, 2015.

12. Singh M, Singh P, Singh S, Juneja PK and Kaur T: Vitamin D receptor (VDR) gene polymorphism influences the risk of osteoporosis in postmenopausal women of Northwest India. Arch Osteoporos 8: 147, 2013.

13. Kitjaroentham A, Hananantachai H, Phonrat B, Preutthipan S and Tungtrongchitr R: Low density lipoprotein receptor-related protein 5 gene polymorphisms and osteoporosis in Thai menopausal women. J Negat Results Biomed 15: 16, 2016.

14. Cvijetic S, Grazio S, Kosovic P, Uremovic M, Nemcic T and Bobic J: Osteoporosis and polymorphisms of osteoprotegerin gene in postmenopausal women-a pilot study. Reumatologia 54: $10-13,2016$

15. Terashima M, Tange S, Ishimura A and Suzuki T: MEG3 Long Noncoding RNA contributes to the epigenetic regulation of epithelial-mesenchymal transition in lung cancer cell lines. J Biol Chem 292: 82-99, 2017.

16. Rajpathak SN, Vellarikkal SK, Patowary A, Scaria V, Sivasubbu S and Deobagkar DD: Human 45,X fibroblast transcriptome reveals distinct differentially expressed genes including long noncoding RNAs potentially associated with the pathophysiology of Turner syndrome. PLoS One 9: e100076, 2014.

17. Wang Q, Li Y, Zhang Y, Ma L, Lin L, Meng J, Jiang L, Wang L, Zhou $\mathrm{P}$ and Zhang Y: LncRNA MEG3 inhibited osteogenic differentiation of bone marrow mesenchymal stem cells from postmenopausal osteoporosis by targeting miR-133a-3p. Biomed Pharmacother 89: 1178-1186, 2017. 
18. Wang L, Han S, Jin G, Zhou X, Li M, Ying X, Wang L, Wu H and Zhu Q: Linc00963: A novel, long non-coding RNA involved in the transition of prostate cancer from androgen-dependence to androgen-independence. Int J Oncol 44: 2041-4449, 2014.

19. Mercer TR, Dinger ME and Mattick JS: Long non-coding RNAs: Insights into functions. Nat Rev Genet 10: 155-159, 2009.

20. Costa FF: Non-coding RNAs: New players in eukaryotic biology. Gene 357: 83-94, 2005.

21. Fitzgerald KA and Caffrey DR: Long noncoding RNAs in innate and adaptive immunity. Curr Opin Immunol 26: 140-146, 2014.

22. Liao Q, Liu C, Yuan X, Kang S, Miao R, Xiao H, Zhao G, Luo H, $\mathrm{Bu} \mathrm{D}$, Zhao H, et al: Large-scale prediction of long non-coding RNA functions in a coding-non-coding gene co-expression network. Nucleic Acids Res 39: 3864-3878, 2011

23. Dong R, Jia D, Xue P, Cui X, Li K, Zheng S, He X and Dong K: Genome-wide analysis of long noncoding RNA (lncRNA) expression in hepatoblastoma tissues. PLoS One 9: e85599, 2014.

24. Wang P, Fu H, Cui J and Chen X: Differential lncRNA-mRNA co-expression network analysis revealing the potential regulatory roles of lncRNAs in myocardial infarction. Mol Med Rep 13: 1195-1203, 2016.

25. Fang M, Zhang P, Zhao Y and Liu X: Bioinformatics and co-expression network analysis of differentially expressed lncRNAs and mRNAs in hippocampus of APP/PS1 transgenic mice with Alzheimer disease. Am J Transl Res 9: 1381-1391, 2017.

26. Yang L, Yi K, Wang H, Zhao Y and Xi M: Comprehensive analysis of lncRNAs microarray profile and mRNA-lncRNA co-expression in oncogenic HPV-positive cervical cancer cell lines. Oncotarget 7: 49917-49929, 2016.

27. Wan ZY, Song F, Sun Z, Chen YF, Zhang WL, Samartzis D, Ma CJ, Che L, Liu X, Ali MA, et al: Aberrantly expressed long noncoding RNAs in human intervertebral disc degeneration: A microarray related study. Arthritis Res Ther 16: 465, 2014.

28. Martinez-Herrero S, Larrayoz IM, Ochoa-Callejero L, Fernández LJ, Allueva A, Ochoa I and Martínez A: Prevention of bone loss in a model of postmenopausal osteoporosis through adrenomedullin inhibition. Front Physiol 7: 280, 2016.

29. Montuenga LM, Martínez A, Miller MJ, Unsworth EJ and Cuttitta F: Expression of adrenomedullin and its receptor during embryogenesis suggests autocrine or paracrine modes of action. Endocrinology 138: 440-451, 1997.

30. Uzan B, Villemin A, Garel JM and Cressent M: Adrenomedullin is anti-apoptotic in osteoblasts through CGRP1 receptors and MEK-ERK pathway. J Cell Physiol 215: 122-128, 2008

31. Sun GJ, Guo T, Chen Y, Xu B, Guo JH and Zhao JN: Significant pathways detection in osteoporosis based on the bibliometric network. Eur Rev Med Pharmacol Sci 17: 1-7, 2013.

32. Zhang X, Alnaeeli M, Singh B and Teng YT: Involvement of SOCS3 in regulation of CD $11 \mathrm{c}^{+}$dendritic cell-derived osteoclastogenesis and severe alveolar bone loss. Infect Immun 77 2000-2009, 2009.

33. Pickard MR, Mourtada-Maarabouni M and Williams GT: Long non-coding RNA GAS5 regulates apoptosis in prostate cancer cell lines. Biochim Biophys Acta 1832: 1613-1623, 2013

34. Sun M, Jin F, Xia R, Kong R, Li JH, Xu TP, Liu YW, Zhang EB, Liu XH and De W: Decreased expression of long noncoding RNA GAS5 indicates a poor prognosis and promotes cell proliferation in gastric cancer. BMC Cancer 14: 319, 2014
35. Qiao HP, Gao WS, Huo JX and Yang ZS: Long non-coding RNA GAS5 functions as a tumor suppressor in renal cell carcinoma. Asian Pac J Cancer Prev 14: 1077-1082, 2013.

36. Mourtada-Maarabouni M, Pickard MR, Hedge VL, Farzaneh F and Williams GT: GAS5, a non-protein-coding RNA, controls apoptosis and is downregulated in breast cancer. Oncogene 28 195-208, 2009.

37. Balla B, Kosa JP, Kiss J, Borsy A, Podani J, Takács I, Lazáry A, Nagy Z, Bácsi K, Speer G, et al: Different gene expression patterns in the bone tissue of aging postmenopausal osteoporotic and non-osteoporotic women. Calcif Tissue Int 82: 12-26, 2008.

38. Rodríguez JP, Montecinos L, Ríos S, Reyes P and Martínez J: Mesenchymal stem cells from osteoporotic patients produce a type I collagen-deficient extracellular matrix favoring adipogenic differentiation. J Cell Biochem 79: 557-565, 2000.

39. Hsiao HB, Lin H, Wu JB and Lin WC: Kinsenoside prevents ovariectomy-induced bone loss and suppresses osteoclastogenesis by regulating classical NF- $\kappa \mathrm{B}$ pathways. Osteoporos Int 24: 1663-1676, 2013.

40. de Castro LF, Lozano D, Portal-Núñez S, Maycas M, De la Fuente M, Caeiro JR and Esbrit P: Comparison of the skeletal effects induced by daily administration of PTHrP (1-36) and PTHrP (107-139) to ovariectomized mice. J Cell Physiol 227: 1752-1760, 2012

41. McKiernan PJ, McElvaney NG and Greene CM: SLPI and inflammatory lung disease in females. Biochem Soc Trans 39: 1421-1426, 2011.

42. Park JJ, Bae CS, Choi BD, Jeong SJ, Wang G, Lim DS, Kim BO, Cho YS, Kim SJ and Jeong MJ: Induction of secretory leukocyte protease inhibitor (SLPI) in estradiol valerate (EV) induced polycystic ovary. Arch Pharm Res 34: 1389-1397, 2011.

43. Al-Daghri NM, Aziz I, Yakout S, Aljohani NJ, Al-Saleh Y, Amer OE, Sheshah E, Younis GZ and Al-Badr FB: Inflammation as a contributing factor among postmenopausal Saudi women with osteoporosis. Medicine (Baltimore) 96: e5780, 2017.

44. Draijer C, Hylkema MN, Boorsma CE, Klok PA, Robbe P, Timens W, Postma DS, Greene CM and Melgert BN: Sexual maturation protects against development of lung inflammation through estrogen. Am J Physiol Lung Cell Mol Physiol 310: L166-L174, 2016.

45. Chen D, Xu X, Cheon YP, Bagchi MK and Bagchi IC: Estrogen induces expression of secretory leukocyte protease inhibitor in rat uterus. Biol Reprod 71: 508-514, 2004.

46. Meyerson HJ, Osei E, Schweitzer K, Blidaru G, Edinger A and Balog A: CD177 expression on neutrophils: In search of a clonal assay for myeloid neoplasia by flow cytometry. Am J Clin Pathol 140: 658-669, 2013.

47. Lattrich C, Schüler S, Häring J, Skrzypczak M, Ortmann O and Treeck O: Effects of a combined treatment with tamoxifen and estrogen receptor $\beta$ agonists on human breast cancer cell lines. Arch Gynecol Obstet 289: 163-171, 2014. 